\title{
Hardware Implementation of COTS Avionics System on Unmanned Aerial Vehicle Platforms
}

\author{
Yoo-Hsiu Yeh ${ }^{1}$ \\ Carnegie Mellon University Silicon Valley, Moffett Field, CA, 94035 \\ Parth Kumar ${ }^{2}$ \\ Polytechnic Institute of New York University, Brooklyn, NY 11201 \\ Abraham Ishihara ${ }^{3}$ \\ Carnegie Mellon University Silicon Valley, Moffett Field, CA, 94035 \\ and \\ Corey Ippolito ${ }^{4}$ \\ NASA Ames Research Center, Moffett Field, CA, 94035
}

\begin{abstract}
Unmanned Aerial Vehicles (UAVs) can serve as low cost and low risk platforms for flight testing in Aeronautics research. The NASA Exploration Aerial Vehicle (EAV) and Experimental Sensor-Controlled Aerial Vehicle (X-SCAV) UAVs were developed in support of control systems research at NASA Ames Research Center. The avionics hardware for both systems has been redesigned and updated, and the structure of the EAV has been further strengthened. Preliminary tests show the avionics operate properly in the new configuration. A linear model for the EAV also was estimated from flight data, and was verified in simulation. These modifications and results prepare the EAV and X-SCAV to be used in a wide variety of flight research projects.
\end{abstract}

\section{Introduction}

$\mathrm{T}$ HE NASA Exploration Aerial Vehicle Lab operates two instrumented commercial, off-the-shelf aerial platforms for testing novel control systems: the Exploration Aerial Vehicle (EAV) and the Experimental SensorControlled Aerial Vehicle (X-SCAV). The EAV is an eight-foot wingspan quarter-scale Cessna 182 that serves as the primary test platform for control systems. The EAV lab currently fields three of these aircraft. The X-SCAV is an 18-foot wingspan half-scale J3 Piper Cub, a much larger platform that can carry payloads up to fifty pounds. Both are low-cost, easy to maintain, FAA compliant, and hence ideal solutions for rapid and low-cost flight testing.

Previous research projects carried out with these platforms include:

1) Testing of the NASA Ames Intelligent Flight Controller (IFC), as detailed in Ref.1

2) Testing of the NASA Ames Polymorphic Control Systems (PCS) controller, as detailed in Ref. 2

3) Simulation testing of the Trajectory Linearization Controller (TLC) in conjunction with Payload Directed Flight (PDF), as detailed in Ref. 3

Both EAV and X-SCAV are outfitted with the same avionics hardware and software architecture, allowing for parallel development. The avionics hardware is continually upgraded to provide state-of-the-art processing power and sensors needed to carry out respective tests.

This paper first provides an update on the latest avionics hardware for the two platforms, covering the requirements followed for the design of the enclosure and integration of components. The second part covers the

\footnotetext{
${ }^{1}$ Project Engineer, Carnegie Mellon Silicon Valley, NASA Ames Research Park, AIAA Member.

${ }^{2}$ Student, Polytechnic Institute of New York University, AIAA Member.

${ }^{3}$ Research Scientist, Carnegie Mellon Silicon Valley, NASA Ames Research Park, AIAA Member.

${ }^{4}$ Research Scientist, Intelligent Systems Division, NASA Ames Research Center, AIAA Member.
} 
system identification of a linear model of the EAV using ordinary least squares, based on previous flight data. The final part covers simulation results of the linear model in MATLAB.
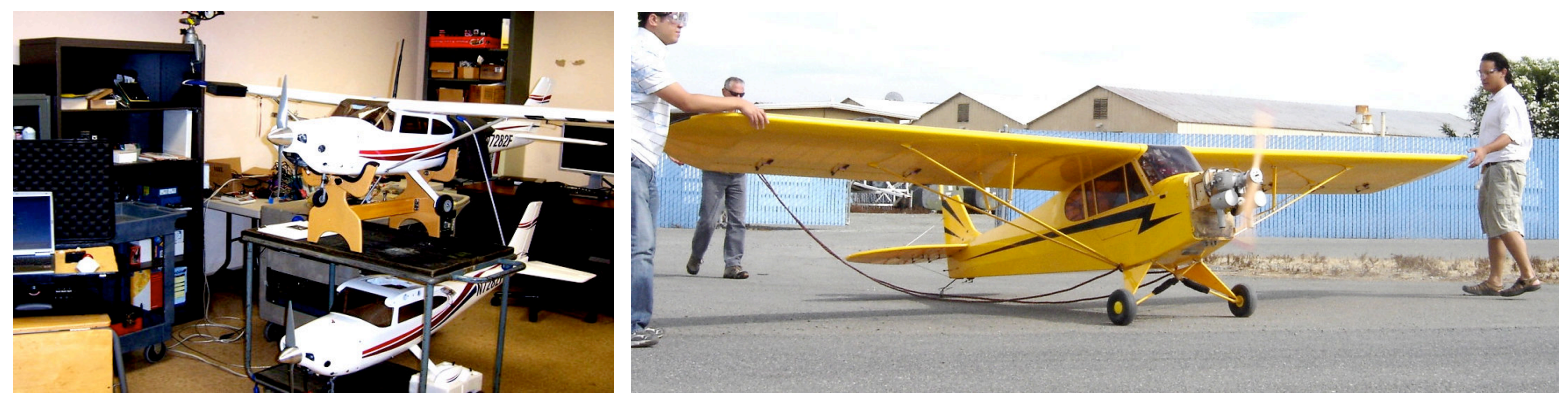

Figure 1. NASA EAVs (left) and X-SCAV (right).

\section{Avionics Design}

In the previous system, the EAV avionics were built around a single onboard computer that logged sensor data, handled data telemetry, and ran the control software. The complete avionics system, including software, data telemetry, servo control, and redundancy, is described in detail in Ref. 1 and is briefly summarized in Figure 2 below.

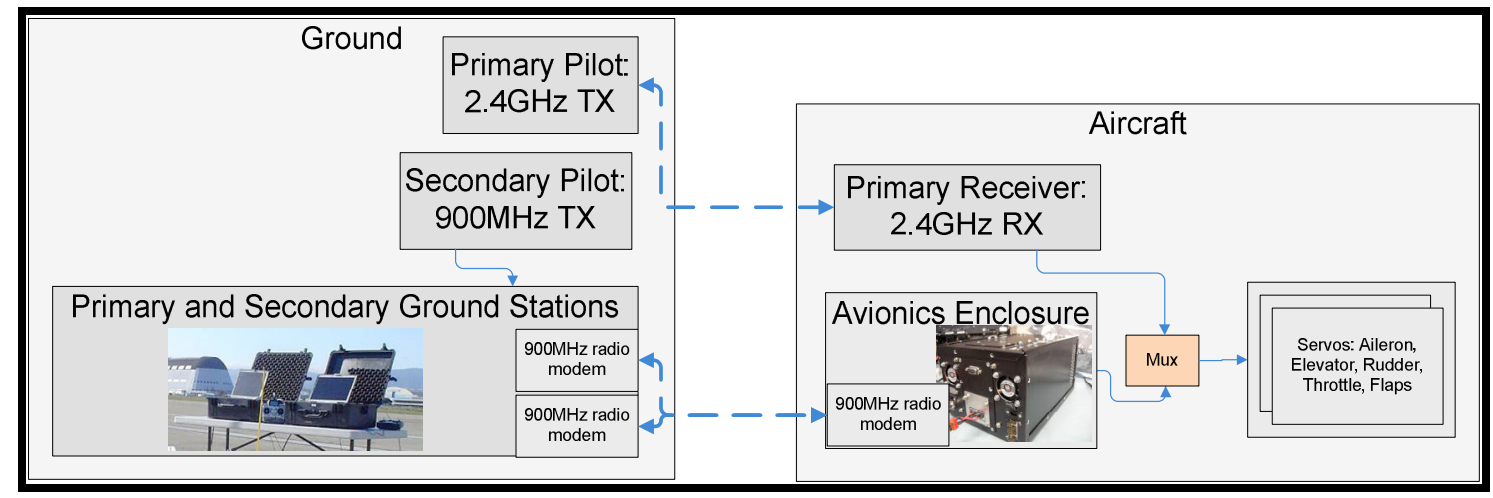

Figure 2. Overview of Ground and Aircraft System Diagram.

For the Payload Directed Flight project, there was a potential need to process large amounts of sensor data quickly, such as image processing for camera images, which is further explained in Refs. 4 and 5. Running the sensor processing on the same unit as the control system could interfere with the main control system. The addition of a second, faster CPU to the avionics stack motivated the redesign of the avionics and enclosure to provide flexibility for future modules.

\section{A. Redesign of Avionics System}

Many of the avionics components for the new system carried over from or were newer models of the previous components. All avionics components were chosen to be ruggedized and vibration-tolerant. 
Table 1. Avionics System Components

\begin{tabular}{|c|c|c|c|}
\hline Component & Manufacturer & Model & Description \\
\hline Power Supply & Tri-M Systems & HPSC104-SER & $\begin{array}{l}\text { 168W DC/DC High Power Ve- } \\
\text { hicle Power Supply }\end{array}$ \\
\hline Servo Controller & Pontech & HBC101 & $\begin{array}{l}\mathrm{A} / \mathrm{D} \text { and hybrid motor con- } \\
\text { troller board }\end{array}$ \\
\hline $\begin{array}{l}\text { Hard } \quad \text { Drive } \\
\text { (ground test only) }\end{array}$ & Western Digital & Scorpio & $\begin{array}{l}\text { 160GB EIDE Notebook Hard } \\
\text { Drive }\end{array}$ \\
\hline CF Cards & Sandisk & Extreme III CF, 8GB & Two (2x) 8GB CFcards \\
\hline $\mathrm{CPU}$ & Advanced Digital Logic & ADL945PC-L2400 & $\begin{array}{l}\text { DualCore } 1.66 \mathrm{GHz}, 2 \mathrm{M} \text { cache } \\
\mathrm{PC} / 104\end{array}$ \\
\hline RTOS CPU & Digital Logic & MSMP3SEV & $\begin{array}{l}\text { SmartCore } \mathrm{P} 3-700,700 \mathrm{MHz} \text {, } \\
256 \mathrm{MB}\end{array}$ \\
\hline $\mathrm{CPU} \mathrm{I} / \mathrm{O}$ & Parvus & COM-1274 & $\begin{array}{l}\text { 8-Port Serial Module with } \\
\text { CAN } 2.0\end{array}$ \\
\hline INS/GPS & Athena Controls, Inc. & Guidestar GS111m & $\begin{array}{l}\text { Digital IMU/INS/GPS Sensor } \\
\text { Suite }\end{array}$ \\
\hline Radio Modem & Microhard System, Inc. & MHX-910 & $\begin{array}{l}\text { ISM Band 900Mhz RF Radio } \\
\text { Modem }\end{array}$ \\
\hline Fan & (Unknown) & (Unknown) & Cooling Fan \\
\hline
\end{tabular}

The three components that were not in the PC/104 form factor were modified to fit in the PC/104 stack. Adapter plates were designed for the $\mathrm{HBC} 101$ servo controller and the compact flash card readers. A custom PCB was designed to serve as the base and electrical interface for the MHX-910 radio modem. This board replaced the default evaluation board, providing power and a RS232-to-TTL interface to the modem.

The five main requirements for the avionics system design were addressed as follows:

1) Avoid ground loops and keep noise from passing between systems.

The overall aircraft had three electrical systems: avionics, actuators, and engine. For the most part, the systems had no overlap, with separate power supplies and grounding. The exception was the servo controller board, which translated commands from the CPU into PWM signals for the actuators. The grounds for the two systems were kept separate by optically isolating the PWM signals outside the avionics enclosure.

The enclosure base was chosen as the ground plane for the avionics electrical system. Components inside the enclosure emit a wide range of high frequencies, from the radio modem leakage at around $900 \mathrm{MHz}$, to the Dual Core CPU at $1.66 \mathrm{GHz}$. Thus, a multi-point grounding system was chosen. The standard singlepoint grounding reduces the noise from ground loops, but at frequencies above $1 \mathrm{MHz}$, cables behaving as antennas due to differences in ground potential are likely to be a larger source of noise (Ref. 6). The stack was grounded to the enclosure at three main points: at either end and also at the center.

Outside the box, the battery ground and ground plane were connected at a single point, as close as possible to where the power cables entered the enclosure (Ref. 7). It is important to note that the enclosure was not used as the power return path. The Tri-M power supply has input filtering, so filters were not used for the power leads.

2) Cables must be bundled to minimize strain and contact with other components, while keeping noise carried between boards by cables to a minimum.

The components were arranged in the box to minimize cable length while keeping components that generated heat apart from each other. In addition, cables were twisted or shielded wherever possible. Both arrangements reduce differential-mode current radiation and magnetic coupling within the box by reducing the enclosed area of the circuit path. Power cables were grouped separately from signal lines, to prevent signal coupling noise, or crosstalk (Ref. 8).

Passing cables through apertures in a shielded enclosure can make the shield completely ineffective because cables can re-radiate as antennas. Thus, shielding of cables that entered the enclosure was also essential to reducing noise. Multiple grounding configurations exist for tying shielded cables to enclosures, but it is not always predictable which will work the best (see Ref.9). For this enclosure, a single-point shield ground configuration was chosen, with the drain wires of shielded cables were pigtailed to ground on one side only, as shown in Figure 3.

3) Avionics components must operate properly in a typical operating EMI environment. 
Installation tests for proper operation were performed, and are briefly outlined in Section $\mathbf{C}$.

4) All wires must be rated to carry their maximum projected current.

For each of the custom cables, the wire gauge was chosen based on safe temperature rise under the maximum expected current load. The equation and tables used to calculate the smallest wire gauge are given in the appendix.

5) All fasteners and cables must have secondary securing mechanism for vibration.

All custom connectors have secondary latching mechanisms. If existing connectors did not have secondary latching, a layer of RTV silicone rubber was applied around the connection to serve as secondary reinforcement. RTV was also over applied over screw terminal connectors to eliminate the possibility of loose screws due to vibration.
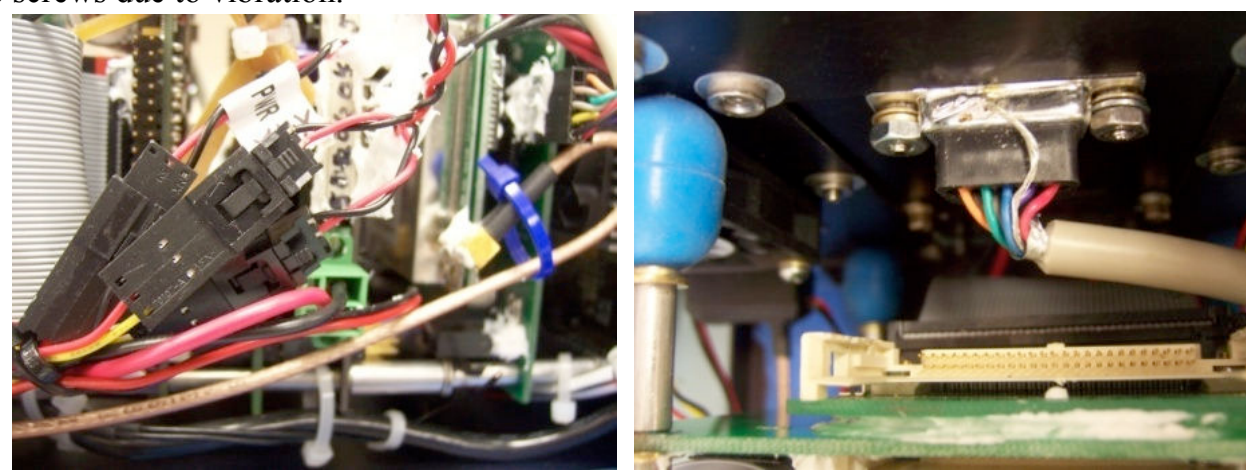

Figure 3. Example of Secondary Latching Mechanisms (left), and Grounded Shielded Cabling (right).

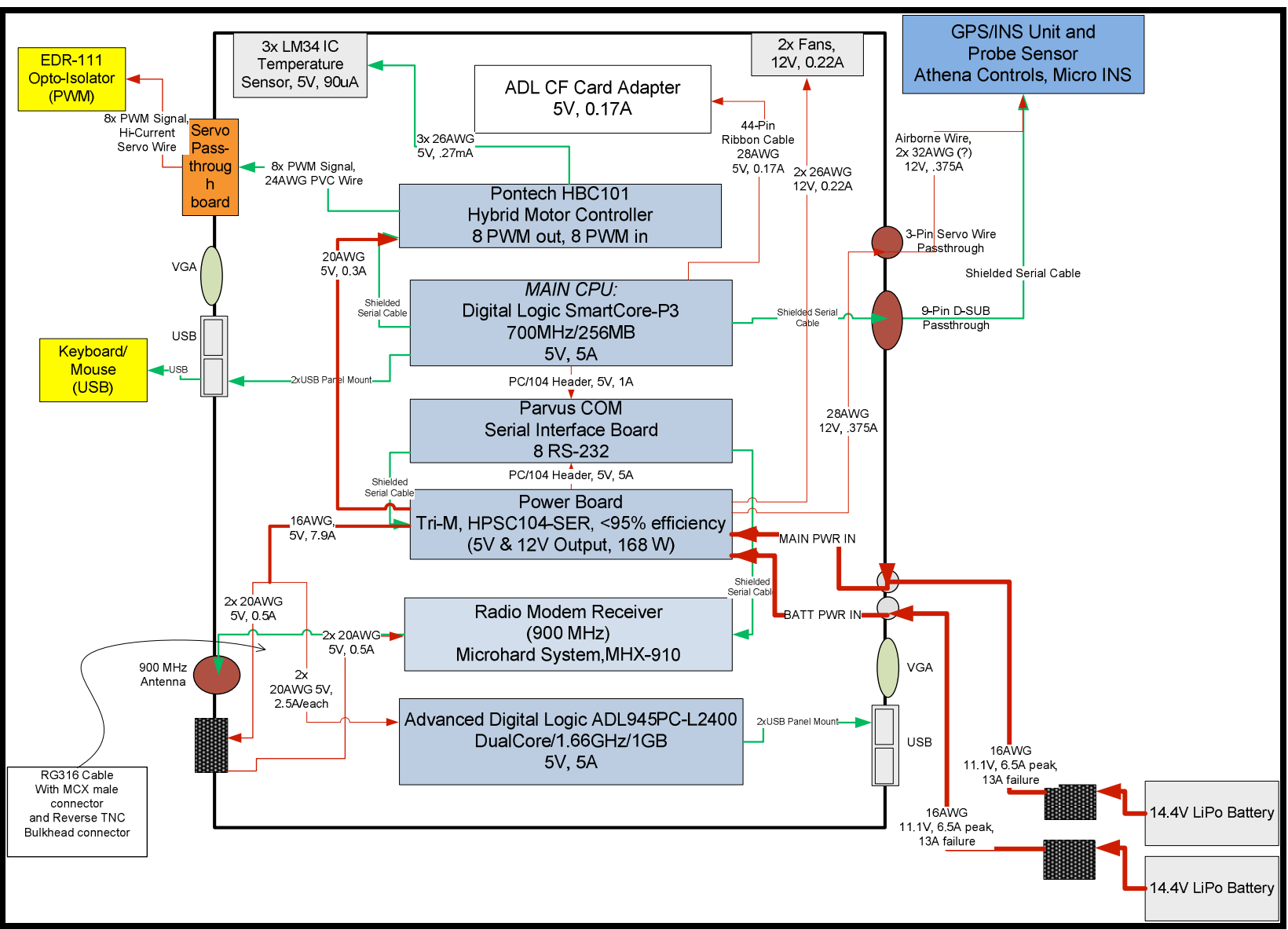

Figure 4. Minimizing Power and Communications Wiring inside the Avionics Box. 


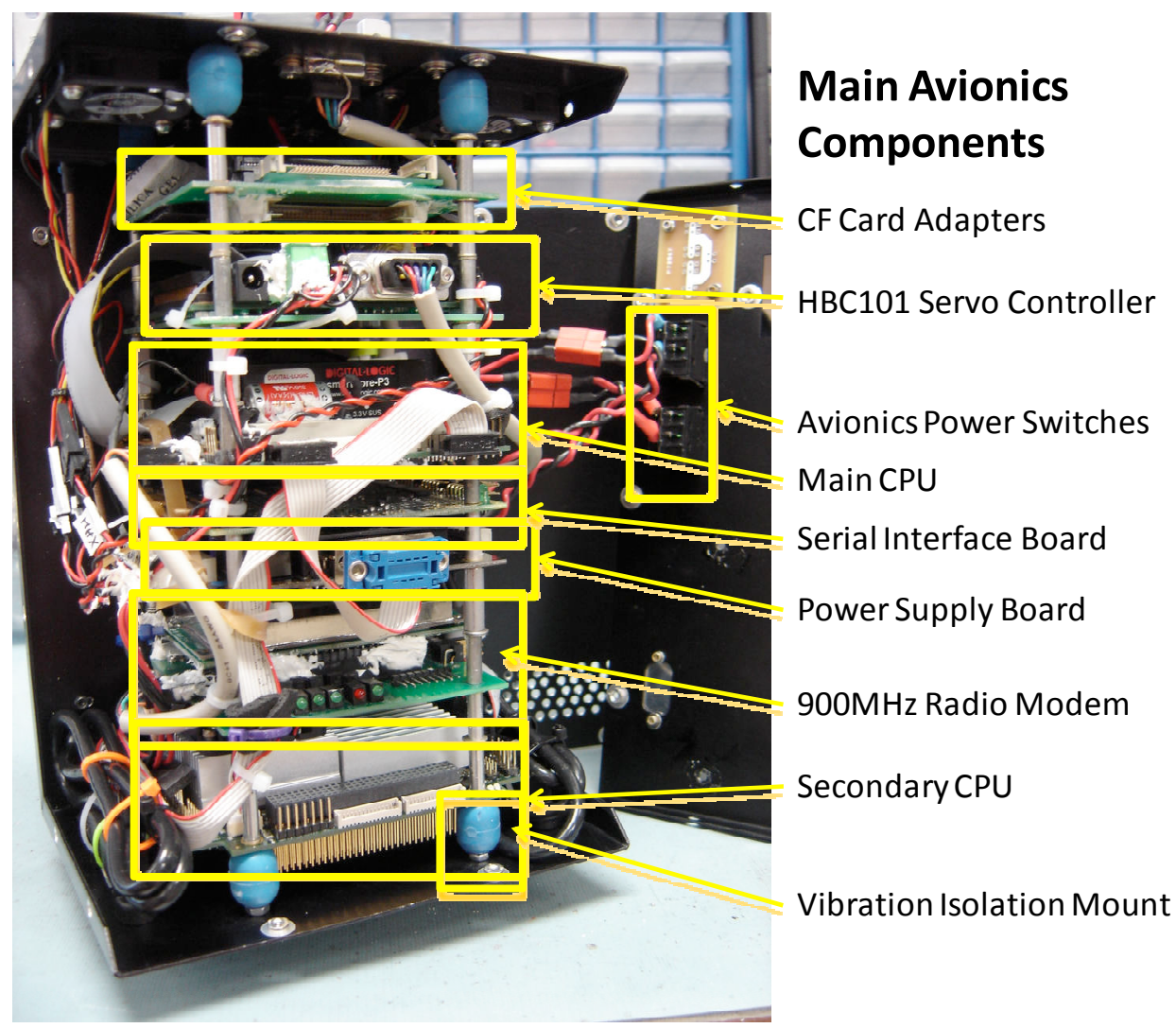

Figure 5. Actual Avionics Box Configuration.
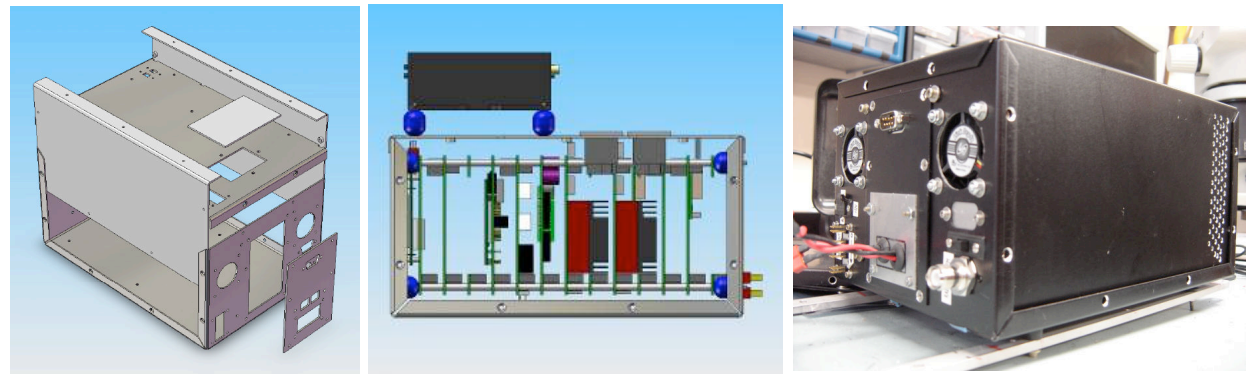

Figure 6. Final Avionics Box Design in Solidworks (left, middle) and Actual (right).

\section{B. Design of the Avionics Enclosure}

The five main requirements for the avionics enclosure design were addressed as follows:

1) Must shield internal components from outside radiation and keep inside radiation from affecting pilot control receiver.

A metal enclosure ensures that noise from avionics components does not affect the key actuator receiver signal, and that noise from the outside environment does not affect the operation of the avionics components. Due to the relatively low cost of the vehicle and available materials, EMI shielding was optimized as much as possible, but not to the degree of full-scale aircraft specifications.

The final box design was bent from 0.040" aluminum. Both inner and outer surfaces were anodized to provide insulation against stray wires. To minimize the number of open edges, the bottom was designed as a single U-shaped piece. The two sides slide out without affecting any other components, allowing connectors on the top to remain attached during ground testing, as can be seen in the left image in Figure 6. To attenuate EMI at the joints, every edge had an overlapping flange. This L-shaped flange forces waves to reflect at least twice before entering the box. Two attachment screws are used per edge to reduce the width of gap openings. Anodization on the box around attachment points was removed to ensure solid electrical connection. 
To approximate the radiating source as a planar wave, the aperture must be outside the near field. The near field of a radiating source is calculated as:

$$
\text { near field radius }=\frac{2\left(D_{\text {antenna }}\right)^{2}}{\lambda}
$$

Where $D_{\text {antenna }}$ is the dimension of the radiating antenna, and $\lambda$ is the wavelength of interest. Thus at distances farther than $16.7 \mathrm{~cm}$ for $900 \mathrm{MHz}$ radiated from a half-wave antenna, and $5.76 \mathrm{~cm}$ for $2.4 \mathrm{GHz}$ radiated from a half-wave antenna, the wave can be approximated as planar. The attenuation of planar waves with wavelengths greater than twice the aperture size through apertures in an enclosure can be described by:

$$
\text { signal attenuation }=20 \log _{10}\left(\frac{2 d_{\text {aper }}}{\lambda}\right)
$$

Where $d_{\text {aper }}$ is the largest dimension of the aperture, and $\lambda$ is the wavelength of interest.

Both eqs. (1) and (2) can be found in Ref. 9, and were used to derive the values in the table below.

Table 2. Signal Attenuation from Apertures in Enclosure.

\begin{tabular}{|c|c|c|c|}
\hline Aperture & $\begin{array}{c}\text { Maximum } \\
\text { Dimension } \\
(\mathbf{c m})\end{array}$ & $\begin{array}{c}\text { Signal } \\
\text { Attenuation at } \\
\mathbf{9 0 0 M h z}(\mathbf{d B})\end{array}$ & $\begin{array}{c}\text { Signal } \\
\text { Attenuation at } \\
\mathbf{2 . 4 G H z}(\mathbf{d B})\end{array}$ \\
\hline Ventilation holes & 0.55 & -68.0 & -48.6 \\
\hline Fan outlets (2) & 4.9 & -24.3 & -4.87 \\
\hline Power line passthroughs (2) & 1.5 & -47.9 & -28.5 \\
\hline Power switch mounts (2) & 3.7 & -29.9 & -10.5 \\
\hline
\end{tabular}

2) Must hold standard PC/104 boards, allowing extra space for standard connectors off the sides as specified in the PC/104 standard.

The PC/104 standard allows components to be stacked together in a four-point mounting system. In order to compensate for the height of the additional CPU in the limited space in the EAV payload bay, it was decided to stack the components horizontally in the enclosure. While the current components only occupy nine spaces, the flexibility to add additional components was designed into the enclosure as well.

The box currently can hold up to thirteen PC/104 boards with the clearances specified in the standard. An extra inch on both sides, and half an inch on top and bottom, allow room for connectors that extend off the board edges, as specified in the standard.

Table 3. Avionics Box Dimensions.

\begin{tabular}{|c|c|}
\hline \multicolumn{2}{|c|}{ Box Specifications } \\
\hline Length & 9.44 in. $(23.98 \mathrm{~cm})$ \\
\hline Width & 6.30 in. $(16.00 \mathrm{~cm})$ \\
\hline Height & 4.78 in. $(12.14 \mathrm{~cm})$ \\
\hline Weight & 5 lbs. 10 oz. $(2.551 \mathrm{~kg})$ \\
\hline
\end{tabular}

3) Must provide vibration isolation to avionics components.

The enclosure mounts to rails in the EAV and to six mounting points in the X-SCAV. In both configurations, the box is mounted to rails via vibration isolation mounts. Vibration isolation is also handled inside the box, where the avionics stack is attached to the box on both ends by Parvus Bumper Beans, as shown in Figure 5.

4) Must be self-contained, and connectors must be easily accessible when installed in aircraft.

The slot on the top of the box was left for ease of swapping out the compact flash cards used for logging data, without having to disassemble the box after every flight test. The center of the rear panel can be swapped out for different connectors as needed. Inside the box behind the points of attachment, type FE self-clinching PEM ${ }^{\circledR}$ miniature fasteners were pressed into the metal instead of nuts to reduce weight and remove the need to reach inside the box.

In the EAV configuration, the front of the box is directly adjacent to the EAV gas tank, so battery connectors are on the rear panel.

5) Must provide sufficient cooling for internal components to operate. 
Five rows of small ventilation holes were drilled along the front end of either side panel. Two $+12 \mathrm{~V}$ CPU fans on the back pull air from the front into the box, drawing heat away from the box components. The HSPC104-SER is a switching power supply with up to $95 \%$ efficiency, so while it may generate some heat, the main source of heat will be the ADL945PC CPU, which draws an average of two to three amps during regular operation. The $\mathrm{CPU}$ is rated for temperatures up to $60^{\circ} \mathrm{C}$, or $140^{\circ} \mathrm{F}$.

\section{Electrical and Mechanical Testing}

Prior to flight testing, the avionics and actuator hardware are taken through a series of functional tests. These are performed with all systems powered on. Tests include:

1) Implementation Testing: Ensure each system can be individually powered and tested. After installations, turn on one system at a time and test to ensure functionality. Once all systems are tested, engage the full system and ensure proper functionality and operation.

2) Ground Vibration Testing: With engine-on, three-point ground contact, run systems for full battery cycle or fuel tank cycle. Check for loose items or damage.

3) Communications and Range Testing: Identify interference to either the $2.4 \mathrm{GHz}$ Primary Radio Control link or $900 \mathrm{MHz}$ Secondary Control link by checking maximum functional range of both control links. Passes if it meets the $100 \mathrm{ft}$ range with $2.4 \mathrm{GHz}$ transmitter at low power mode, and maximum flight box distance at $900 \mathrm{MHz}$ link, which may be up to $1000 \mathrm{ft}$.

The avionics enclosure has passed tests (1) and (2), showing that components are functional under the vibration and temperature environments. Test (3) will be carried out at a future time.

\section{EAV Airframe Modifications}

Previous papers (see Ref. 1) have documented the effects of stress on the EAV airframe. High-g maneuvers have resulted in snapping of the wing struts and failure of the fuselage strut mounts, and the added weight of the avionics box reduces the maneuverability of the aircraft. In preparation for the new avionics box, which will be about a pound heavier than the previous box, and also to prepare for the possibility of future high-g maneuvers, a custom set of aluminum ribs was designed, which are shown in Figure 7.

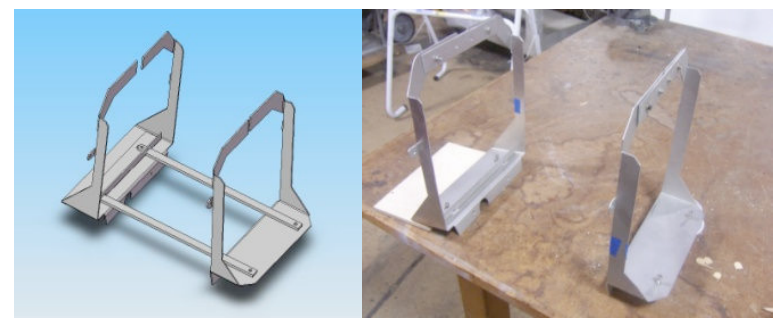

Figure 7. Ribs to Strengthen EAV Structure.

The ribs distribute the stress between the six areas of the EAV airframe with the heaviest load: the wing bar area, from which most the lift of the wings is distributed to the fuselage, the body wing strut mounts, which also distribute some of the stress from the lift, and the landing gear, which pass the impact of hard landings into the belly of the plane.

\section{System Identification of EAV}

Prior system identification of the EAV is documented in Ref. 1 and 2. In those tests, there was difficulty in matching the yaw channel, and this was discovered to be due to an error in the rotation matrix for $p, q$, and $r$. A new system identification process was carried out on the corrected data, and is documented in this section.

The flight data used was from system identification tests carried out over the fall of 2005 and spring of 2006. These included doublets and 3-2-1-1 commands on all control surfaces individually, starting from a trimmed flight condition. A 3-2-1-1 command is a series of high and low pulses with widths in the ratio 3-2-1-1. There were a total of five elevator data sets, five aileron data sets, two rudder data sets, and three throttle data sets chosen for the analysis.

\section{A. Preliminary Data Processing}

A number of functions from the System Identification Programs for Aircraft (SIDPAC) MATLAB software also were used to supplement the system identification process (Ref. 10). The airchk and rotchk functions of the 
SIDPAC software package were used to check the EAV data for compatibility. These functions use a subset of the measured data to reconstruct what values of other measured data should be. The comparison can show biases, drift, or scale factor errors. Drift was observed in roll and pitch measurements, and was removed with the Matlab detrend command.

\section{B. Ordinary Least Squares}

Both nonlinear and linear aircraft models were estimated using Ordinary Least Squares. A quick recapitulation of the Least Squares regression method is below. Consider:

$$
z=X \theta+v
$$

Where:

$$
\begin{aligned}
& z=[z(1) z(2) \ldots z(N)]^{T} \in N \mathrm{x} 1 \text { vector } \\
& \theta=\left[\begin{array}{llll}
\theta_{0} & \theta_{1} & \ldots & \theta_{n}
\end{array}\right]^{T} \in n \mathrm{x} 1 \text { vector of unknown parameters } \\
& X=\left[\begin{array}{lllll}
\xi_{1} & \xi_{2} & \ldots & \xi_{n}
\end{array}\right] \in N \mathrm{x} n \text { matrix of vectors of regressors } \\
& v=[v(1) v(2) \ldots v(N)]^{T} \in N \mathrm{x} 1 \text { vector of measurement errors }
\end{aligned}
$$

The least-squares estimator of $\theta$, denoted as $\hat{\theta}$, is calculated via the following equation:

$$
\hat{\theta}=\left(X^{T} X\right)^{-1} X^{T} z
$$

\section{Linear Aircraft Model}

The EAV was modeled using a linear decoupled aircraft model, as given in Ref. 12.

$$
\begin{array}{r}
{\left[\begin{array}{c}
\Delta \dot{u} \\
\Delta \dot{w} \\
\Delta \dot{q} \\
\Delta \dot{\theta}
\end{array}\right]=\left[\begin{array}{cccc}
X_{u} & X_{w} & 0 & -g \\
Z_{u} & Z_{w} & u_{0} & 0 \\
M_{u}+M_{\dot{w}} Z_{u} & M_{w}+M_{\dot{w}} Z_{w} & M_{q}+M_{\dot{w} u_{0}} & 0 \\
0 & 0 & 1 & 0
\end{array}\right]\left[\begin{array}{c}
\Delta u \\
\Delta w \\
\Delta q \\
\Delta \theta
\end{array}\right]+\left[\begin{array}{cc}
X_{\delta_{e}} & X_{\delta_{T}} \\
Z_{\delta_{e}} & Z_{\delta_{T}} \\
M_{\delta_{e}}+M_{\dot{w}} Z_{\delta_{e}} & M_{\delta_{T}}+M_{\dot{w}} Z_{\delta_{T}} \\
0 & 0
\end{array}\right]\left[\begin{array}{c}
\Delta \delta_{e} \\
\Delta \delta_{T}
\end{array}\right]} \\
{\left[\begin{array}{c}
\Delta \dot{\beta} \\
\Delta \dot{p} \\
\Delta \dot{r} \\
\Delta \dot{\phi}
\end{array}\right]=\left[\begin{array}{cccc}
\frac{Y_{\beta}}{u_{0}} & \frac{Y_{p}}{u_{0}} & -\left(1-\frac{Y_{r}}{u_{0}}\right) & \frac{g \cos \theta_{0}}{u_{0}} \\
L_{\beta} & L_{p} & L_{r} & 0 \\
N_{\beta} & N_{p} & N_{r} & 0 \\
0 & 1 & 0 & 0
\end{array}\right]\left[\begin{array}{c}
\Delta \beta \\
\Delta p \\
\Delta r \\
\Delta \phi
\end{array}\right]+\left[\begin{array}{cc}
0 & \frac{Y_{\delta_{r}}}{u_{0}} \\
L_{\delta_{a}} & L_{\delta_{r}} \\
N_{\delta_{a}} & N_{\delta_{r}} \\
0 & 0
\end{array}\right]\left[\begin{array}{c}
\delta_{a} \\
\delta_{r}
\end{array}\right]}
\end{array}
$$

The variables are defined as:

$u, w$
$p, q, r$
$\beta$
$\theta \phi$
$X_{u}, X_{w}, X_{\delta_{e}}, X_{\delta_{T}}$
$Y_{\beta}, Y_{p}, Y_{r}, Y_{\delta_{r}}$
$Z_{u}, Z_{w}, Z_{\delta_{e}}, Z_{\delta_{T}}$
$L_{\beta}, L_{p}, L_{r}, L_{\delta_{a}}, L_{\delta_{r}}$
$M_{u}, M_{w}, M_{q}, M_{\dot{w}}, M_{\delta_{e}}, M_{\delta_{T}}$
$N_{\beta}, N_{p}, N_{r}, N_{\delta_{a}}, N_{\delta_{r}}$
$\delta_{a}, \delta_{r}, \delta_{e}$
$\delta_{T}$

$u, w$

$p, q, r$

$X_{u}, X_{w}, X_{\delta_{e}}, X_{\delta_{T}}$

$Y_{\beta}, Y_{p}, Y_{r}, Y_{\delta_{r}}$

$Z_{u}, Z_{w}, Z_{\delta_{e}}, Z_{\delta_{T}}$

$L_{\beta}, L_{p}, L_{r}, L_{\delta_{a}}, L_{\delta_{r}}$

$N_{\beta}, N_{p}, N_{r}, N_{\delta_{a}}, N_{\delta_{r}}$

${ }_{a}, \delta_{r}, \delta_{e}$ $x$ and $z$ body-axis velocities of the aircraft relative to Earth axes

body-axis angular velocities

sideslip angle

Euler pitch and roll angles

$\mathrm{X}$-force dimensional derivatives

Y-force dimensional derivatives

Z-force dimensional derivatives

dimensional moment derivatives about the $\mathrm{Y}$ axis

dimensional moment derivatives about the $\mathrm{X}$ axis

dimensional moment derivatives about the $\mathrm{Z}$ axis

control surface deflections in aileron, rudder, and elevator

change in thrust input

The percentage fit of each estimated model to the measured data was calculated as: 


$$
F I T=100 *\left(1-\frac{\|(\text { measured }- \text { estimated }) \|}{\|(\text { measured }- \text { mean }(\text { measured })) \|}\right)
$$

This was based on the FIT equation used in the Matlab System Identification Toolbox. Using the parameters that generated the best fit for each system, the linear model matrices are:

$$
\begin{gathered}
A_{\text {lon }}=\left[\begin{array}{cccc}
-0.0665 & -0.2893 & 0 & -9.81 \\
0.0980 & -1.7836 & -13.1444 & 0 \\
-0.0201 & 0.8228 & -0.6997 & 0 \\
0 & 0 & 1 & 0
\end{array}\right], B_{\text {lon }}=\left[\begin{array}{cc}
4.7690 & 3.1933 \\
28.3783 & 0.1438 \\
-14.5874 & -0.05453 \\
0 & 0
\end{array}\right] \\
A_{\text {lat }}=\left[\begin{array}{cccc}
-0.0523 & 0.0482 & -0.2468 & -5.527 e-03 \\
-19.0019 & -1.6689 & 2.4149 & 0 \\
17.8490 & -0.2100 & -0.8977 & 0 \\
0 & 0.9562 & -0.1630 & 0
\end{array}\right], B_{\text {lat }}=\left[\begin{array}{cc}
0 & -0.3084 \\
-56.5995 & -3.6516 \\
2.1485 & -7.7916 \\
0 & 0
\end{array}\right]
\end{gathered}
$$

Example plots of the best fit data for each parameter are below. The estimated parameters were able to capture the major trends in the data, and damping and frequencies of the modes are similar to those of other UAVs (Ref. 13 and 14).
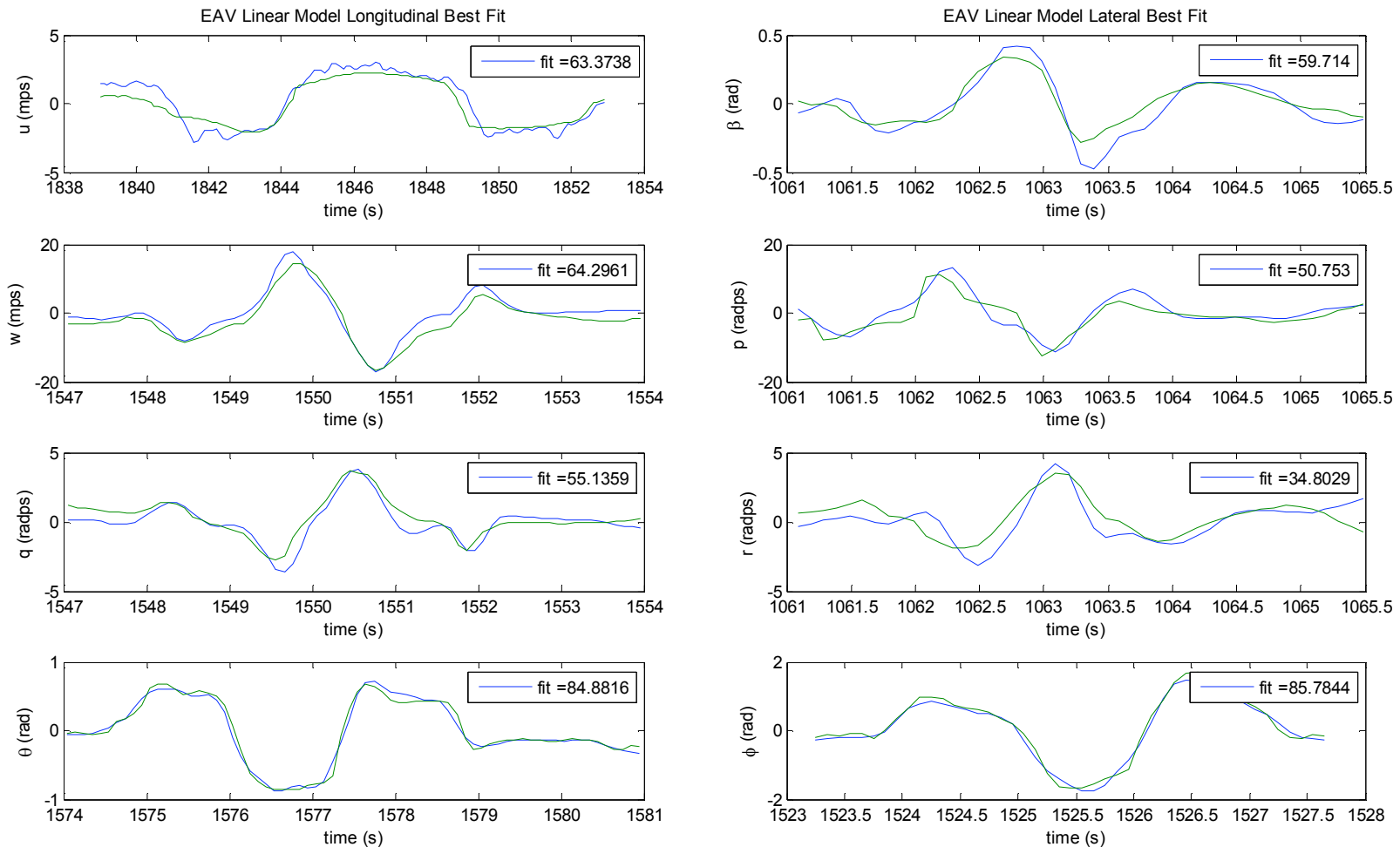

Figure 8. Longitudinal (right) and Lateral (left) Linear Model Fitting.

Longitudinal Modes:

\begin{tabular}{|cccc|}
\hline Mode & Eigenvalue & Damping & Frequency $(\mathrm{rad} / \mathrm{s})$ \\
\hline Phugoid Mode & $-2.41 \mathrm{e}-02+/-3.05 \mathrm{e}-01 \mathrm{i}$ & $7.88 \mathrm{e}-02$ & $3.05 \mathrm{e}-01$ \\
Short Period Mode & $-1.14+/-2.39 \mathrm{i}$ & $4.29 \mathrm{e}-01$ & 2.65 \\
\hline
\end{tabular}


Lateral Modes:

\begin{tabular}{|cccc|}
\hline Mode & Eigenvalue & Damping & Frequency $(\mathrm{rad} / \mathrm{s})$ \\
\hline Spiral Mode & $-1.54 \mathrm{e}-02$ & 1 & $1.54 \mathrm{e}-02$ \\
Roll Mode & -1.21 & 1 & 1.21 \\
Dutch Roll Mode & $-6.95 \mathrm{e}-01+/-2.29 \mathrm{i}$ & $2.90 \mathrm{e}-01$ & 2.39 \\
\hline
\end{tabular}
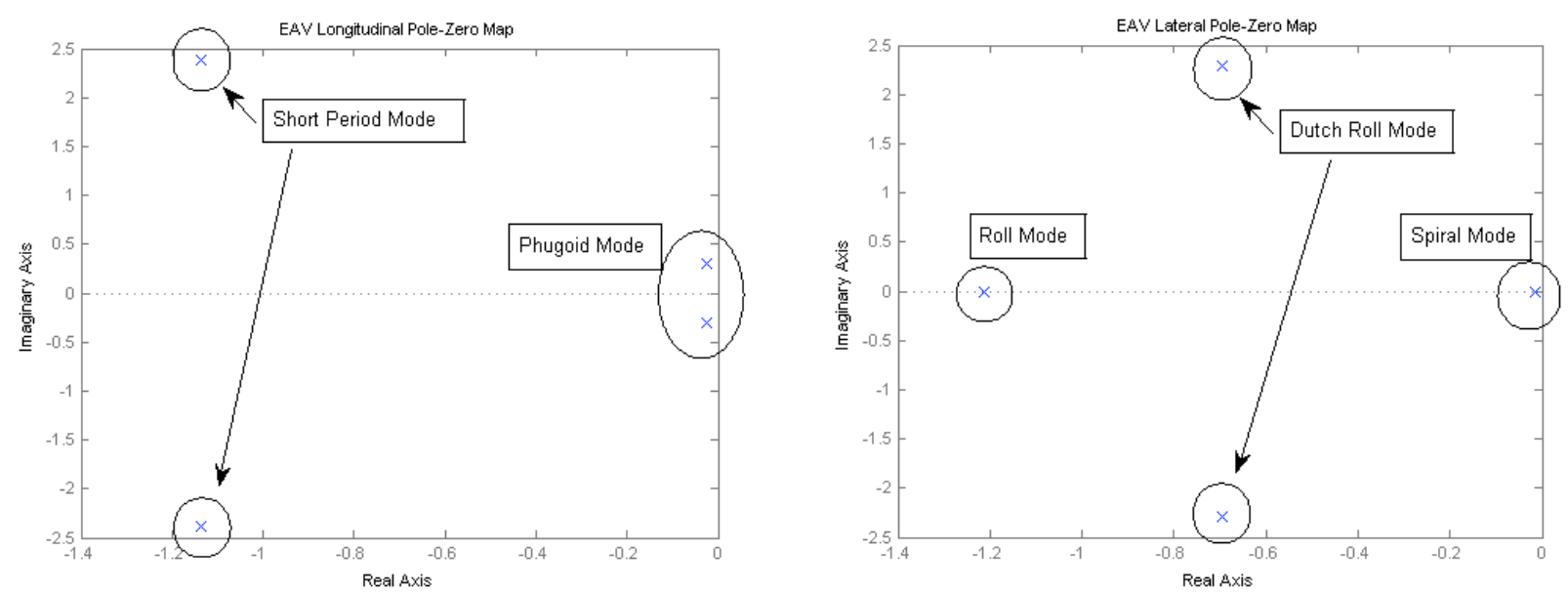

Figure 9. Longitudinal (left) and Lateral (right) Pole-zero plots for EAV.

\section{MATLAB Simulation}

Results from linear simulations of the models are shown below. The Fourth-Order Runge-Kutta numerical integration method was used.

In the longitudinal simulation, the model reflects the trends of the original data. Due to the elevator and throttle input data sets being taken separately, elevator datasets could not be used to derive throttle coefficients, and vice versa. Nonetheless, the best fit parameters show a good match in simulation for both elevator and throttle inputs. This supports that the model adequately includes the modes excited by both longitudinal inputs.
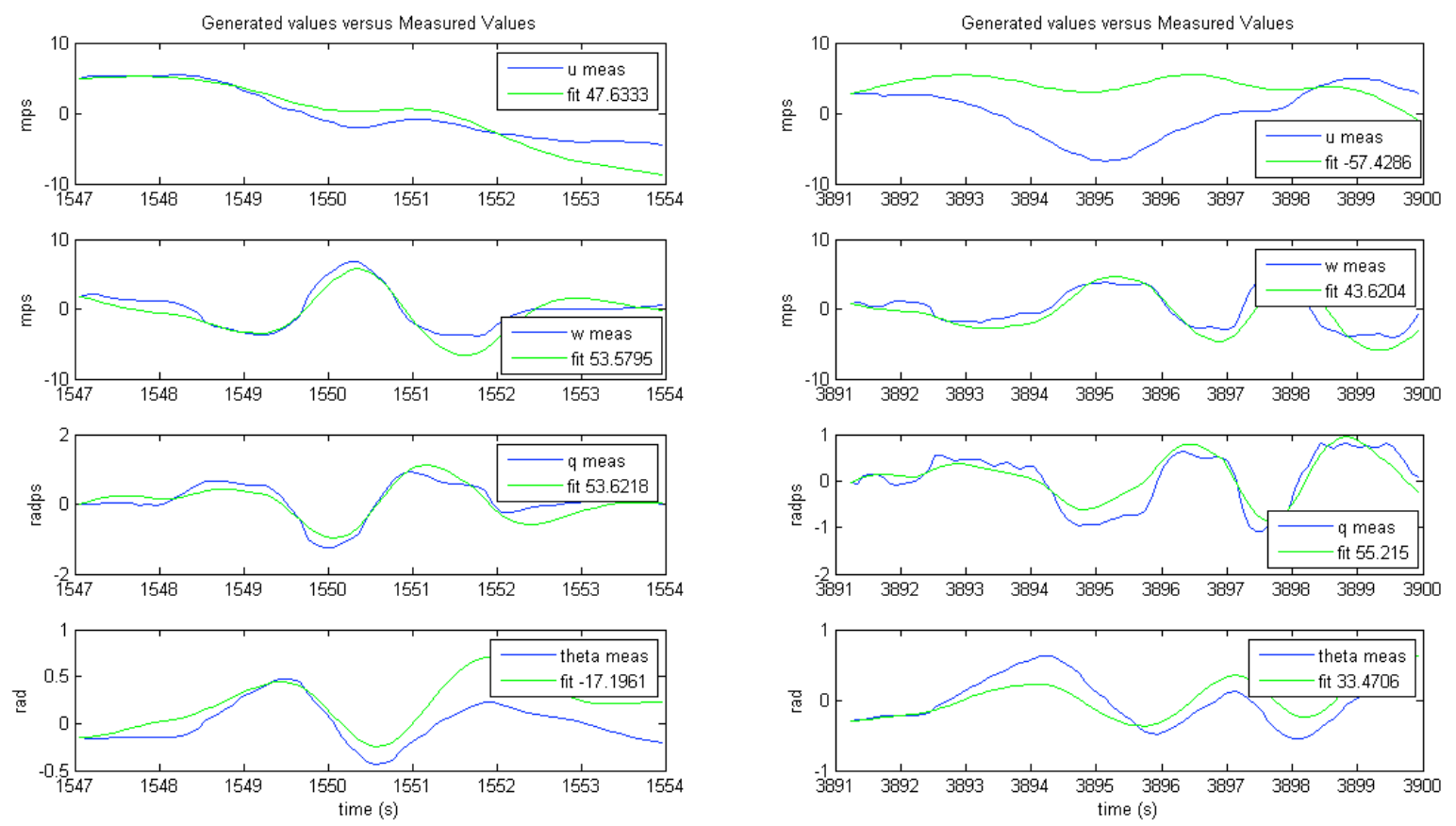

Figure 10. Linear Longitudinal EAV Simulations: Two Elevator Data Sets

10

American Institute of Aeronautics and Astronautics 

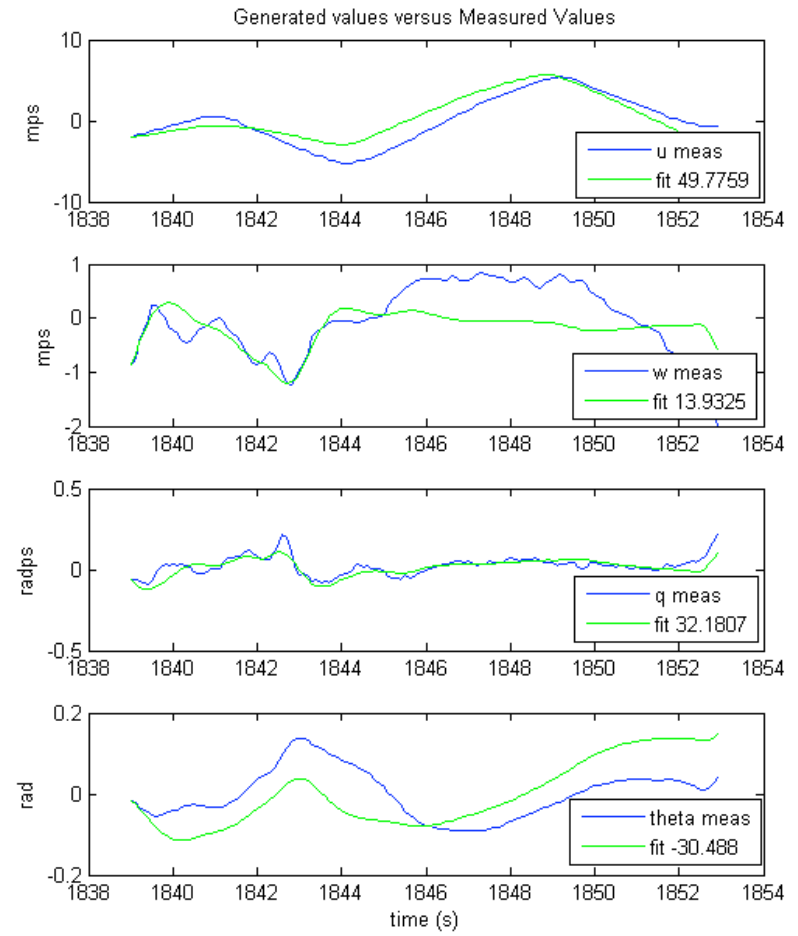

\section{Figure 11. Linear Longitudinal EAV Simulation: Throttle Doublet}

In the lateral model, the simulation reflects the major trends in all channels except the $r$ channel for the rudder data sets, and all channels except the $p$ channel for the aileron data sets. As was the case in the longitudinal data sets, there were no data sets with both aileron and rudder excitation, and thus the dimensional derivatives for the aileron and rudder deflection were estimated from separate data sets. However, unlike the longitudinal simulation, the lateral simulation does not show very good fit matching, despite having good parameter matching as shown in the previous section. In particular, the simulated $p$ channel has almost an inverted fit for the aileron data sets.

This may be due to nonlinearities not captures in the model or excess parameters with little effect that were not removed in the least-squares regression. If it is the second case, a reduced model may remedy this mismatch, and will be derived via step-wise regression in future work. 

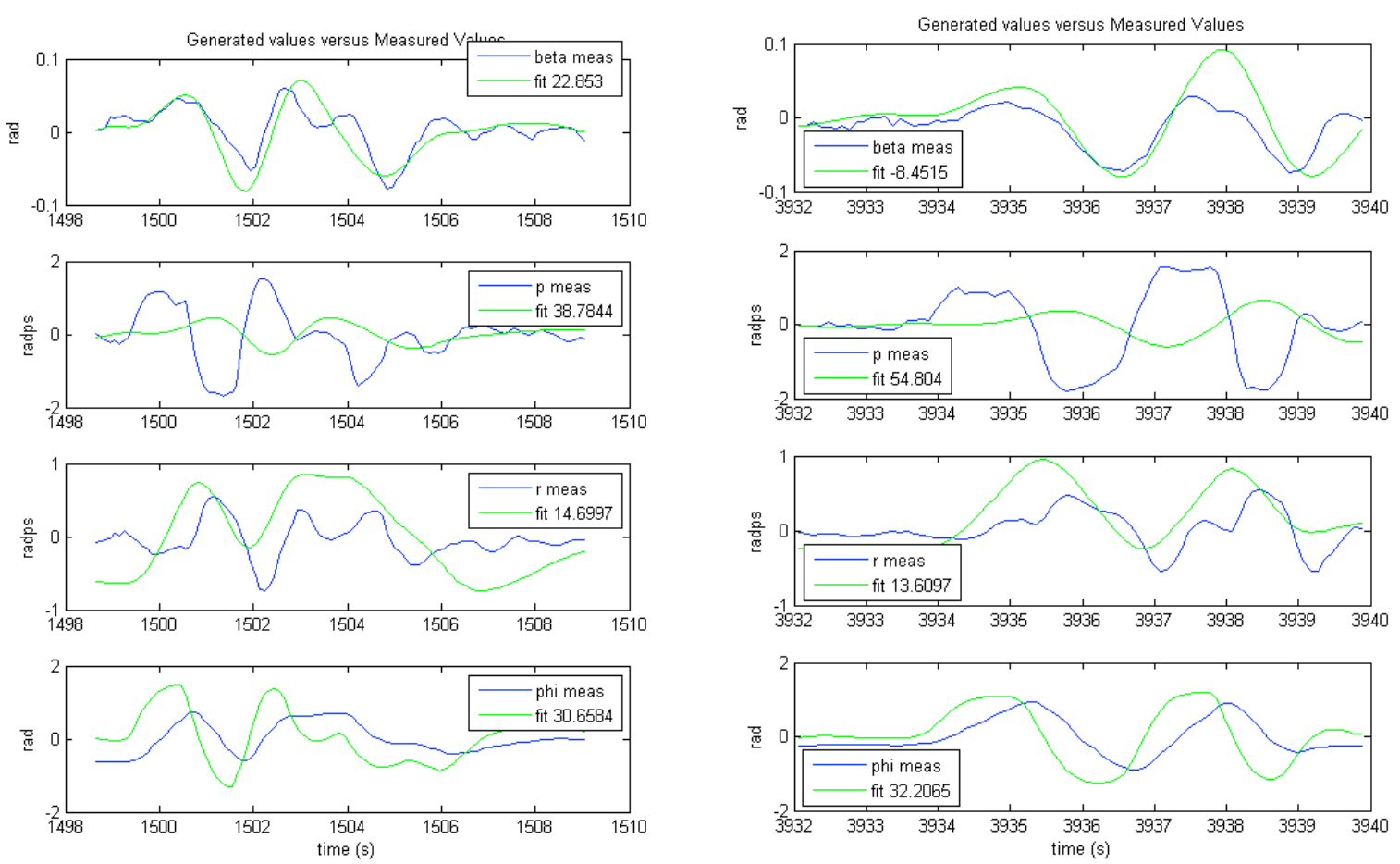

Figure 12. Linear Lateral EAV Simulations: Two Aileron Doublets
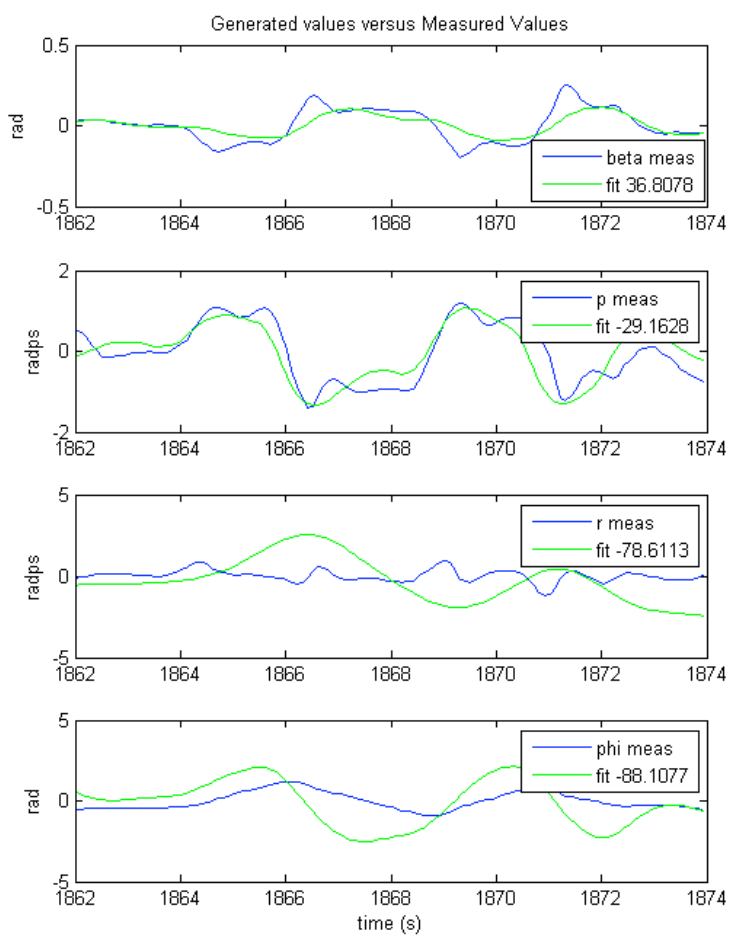

Figure 13. Linear Lateral EAV Simulation: Rudder Doublet 


\section{Conclusion}

This paper reviews the design and testing process for the new EAV/X-SCAV avionics hardware, as well as the system identification of corrected flight data logged on the EAV. These improvements in hardware and aircraft model will help the EAV and X-SCAV continue to serve as low-cost platforms for rapid preliminary low-risk testing of novel controllers.

\section{Appendix}

Determining wire gauge for current draw

For a given current, the wire gauge should be chosen so that heat rise due to resistive losses stays under the rated temperature of the insulation. The majority of the wire used inside the enclosure had PNP Polyvinyl Chloride (PVC) insulation, rated for operation up to 80C. The ribbon cable used for the IDE connection to the CF cards was rated for up to 105C. Servo wire is usually coated in silicone insulation, which can handle up to an estimated 150C.

To find the temperature rise of a material over a given time interval, divide the power loss over that interval by the mass and specific heat capacity, as in the formula below:

$$
T_{f}-T_{i}=\frac{q}{\text { mass } * \text { specific heat }}
$$

Power loss across a length of wire can be calculated by multiplying current by the voltage drop between the two ends. Voltage drop depends on the wire resistance, which is also a function of temperature:

$$
R_{T}=R_{0} *\left(1+\alpha\left(T-T_{0}\right)\right)
$$

Reference tables are included below for copper and aluminum cabling in wire gauge sizes 2 to 36 .

Table 4. Properties of Copper and Aluminum.

\begin{tabular}{|c|ccc|}
\hline Material & $\begin{array}{c}\text { Heat Capacity } \\
(J /(g * K))\end{array}$ & $\begin{array}{c}\text { Density } \\
\left(\mathrm{g} / \mathrm{cm}^{3}\right)\end{array}$ & $\begin{array}{c}\text { Temperature Coefficient of Resistance } \alpha \\
(\Omega / K)\end{array}$ \\
\hline Copper & 0.385 & 8.92 & 0.0039 \\
Aluminum & 0.897 & 2.7 & 0.0039 \\
\hline
\end{tabular}

Table 5. Resistance and Diameter for given Wire Gauge.

\begin{tabular}{|c||c|c|c|c|c|}
\hline AWG & $\begin{array}{c}\text { Diameter } \\
(\text { circ.mils })\end{array}$ & $\begin{array}{c}\text { Diameter } \\
(\mathrm{mm})\end{array}$ & $\begin{array}{c}\text { Area } \\
\left(\mathrm{mm}^{2}\right)\end{array}$ & $\begin{array}{c}\text { Copper Resistance } \\
(\Omega / \mathrm{cm})\end{array}$ & $\begin{array}{c}\text { Aluminum Resistance } \\
(\Omega / \mathrm{cm})\end{array}$ \\
\hline 2 & 521.173 & 6.543 & 33.624 & $4.921 \mathrm{e}-06$ & $8.399 \mathrm{e}-06$ \\
4 & 327.813 & 5.189 & 21.149 & $7.874 \mathrm{e}-06$ & $1.339 \mathrm{e}-05$ \\
6 & 206.120 & 4.115 & 13.298 & $1.542 \mathrm{e}-05$ & $2.126 \mathrm{e}-05$ \\
8 & 129.687 & 3.264 & 8.367 & $2.198 \mathrm{E}-05$ & $3.379 \mathrm{E}-05$ \\
10 & 81.553 & 2.588 & 5.261 & $3.281 \mathrm{E}-05$ & $5.381 \mathrm{E}-05$ \\
12 & 51.276 & 2.052 & 3.308 & $5.577 \mathrm{E}-05$ & $8.563 \mathrm{E}-05$ \\
14 & 32.271 & 1.628 & 2.082 & $8.530 \mathrm{E}-05$ & $1.358 \mathrm{E}-04$ \\
16 & 20.268 & 1.290 & 1.308 & $1.378 \mathrm{E}-04$ & $2.162 \mathrm{E}-04$ \\
18 & 12.756 & 1.024 & 0.823 & $2.165 \mathrm{E}-04$ & $3.445 \mathrm{E}-04$ \\
20 & 8.042 & 0.813 & 0.519 & $3.445 \mathrm{E}-04$ & $5.479 \mathrm{E}-04$ \\
22 & 5.067 & 0.645 & 0.327 & $5.512 \mathrm{E}-04$ & $8.694 \mathrm{E}-04$ \\
24 & 3.173 & 0.511 & 0.205 & $8.957 \mathrm{E}-04$ & $1.381 \mathrm{E}-03$ \\
26 & 1.986 & 0.404 & 0.128 & $1.430 \mathrm{E}-03$ & $2.198 \mathrm{E}-03$ \\
28 & 1.247 & 0.320 & 0.080 & $2.323 \mathrm{E}-03$ & $3.478 \mathrm{E}-03$ \\
30 & 0.785 & 0.254 & 0.051 & $3.707 \mathrm{E}-03$ & $5.545 \mathrm{E}-03$ \\
32 & 0.503 & 0.203 & 0.032 & $5.709 \mathrm{E}-03$ & $8.825 \mathrm{E}-03$ \\
34 & 0.312 & 0.160 & 0.020 & $9.186 \mathrm{E}-03$ & $1.404 \mathrm{E}-02$ \\
36 & 0.196 & 0.127 & 0.013 & $1.460 \mathrm{E}-02$ & $2.234 \mathrm{E}-02$ \\
\hline
\end{tabular}

table data from: http://www.powerstream.com/Wire_Size.htm, http://www.proav.de/index.html?http\&\&\&www.proav.de/data/wireresistance.html, http://www.interfacebus.com/Aluminum_Wire_AWG_Size.html 


\section{Acknowledgments}

Many thanks to all the EAV lab interns who contributed to the design of the first custom avionics box and EAV support ribs: Matthew Walliser, Augustus Shelander, Bradley Jackson, Matthew Wallach, and Mera Horne. The final avionics box design, as described in this paper, was based on the prototypes they developed.

The final support ribs design was done by Don Hineman, with the support of Vince Perry. Vince was also responsible for the original reinforcement of the EAV structure, and his expertise in all things regarding small aircraft is very much appreciated.

Additional thanks go to Dongwon Jung of Georgia Tech, who provided data that allowed us to verify our system identification process.

\section{References}

${ }^{1}$ Ippolito, C., Yeh, YH., and Kaneshige, J. Neural Adaptive Flight Control Testing on an Unmanned Experimental Aerial Vehicle. AIAA-2008-2827. May 2007.

${ }^{2}$ Ippolito, C., Joo, S., Al-Ali, K., and Yeh, YH. Polymorphic Control Reconfiguration in an Autonomous UAV with UGV Collaboration. Aerospace Conference, 2008 IEEE. March 2008.

${ }^{3}$ Adami, T., Zhu, J., Ishihara, A., Yeh, YH, and Ippolito, C. Six DoF Trajectory Tracking for Payload Directed Flight Using Trajectory Linearization Control. AIAA-2009-1897. April 2009.

${ }^{4}$ Ippolito, C., Campbell, S., and Yeh, YH. A Trajectory Generation Approach for Payload Directed Flight. AIAA-20091351. January 2009.

${ }^{5}$ Lee. R. and Ippolito, C. A Perception and Mapping Approach for Plume Detection in Payload Directed Flight. AIAA2009-1898. April 2009.

${ }^{6}$ Van Doren, T. and Hubing, T. EMC Design Guidelines Prepared for Boeing Commercial Avionics Systems. TR92-1-004R. University of Missouri-Rolla, Electromagnetic Compatibility Laboratory. January 15, 1992.

${ }^{7}$ Gnecco, L.T. Design of Shielded Enclosures: Cost-Effective Methods to Prevent EMI. Newnes, 2000.

${ }^{8}$ Horowitz, P. and Hill, W. The Art of Electronics, Second Edition. Cambridge University Press, 1989. p.455-461.

${ }^{9}$ Electromagnetic Compatibility Design Guide for Avionics and Related Ground Support Equipment. NAVAIR AD 1115. $3^{\text {rd }}$ Edition. Naval Air Systems Command. June 1988.

${ }^{10}$ Klein, V. and Morelli, E.A. Aircraft System Identification: Theory and Practice. AIAA, AIAA Education Series. 2006.

${ }^{11}$ Nelson, R. Flight Stability and Automatic Control. $2^{\text {nd }}$ Edition. WCB/McGraw-Hill, 1998.

12 Jung, D. Hierarchical Path Planning and Control of a Small Fixed-Wing UAV: Theory and Experimental Validation. Thesis Presented to Georgia Institute of Technology. December 2007.

${ }^{13}$ Jang, J.S., and Tomlin, C. Longitudinal Stability Augmentation System Design for the DragonFly UAV Using a Single GPS Receiver. AIAA-2003-5592. August 2003. 